\title{
Conway's group and octonions
}

\author{
Robert A. Wilson \\ School of Mathematical Sciences, \\ Queen Mary, University of London, \\ Mile End Road, London E1 4NS
}

Submitted 17th March 2009

\begin{abstract}
We give a description of the double cover of Conway's group in terms of right multiplications by $3 \times 3$ matrices over the octonions. This leads to simple sets of generators for many of the maximal subgroups, including a uniform construction of the Suzuki chain of subgroups.
\end{abstract}

\section{Introduction}

In [12] I showed how to construct a 3-dimensional octonionic Leech lattice, based on Coxeter's non-associative ring of integral octonions [5], which is an algebraic version of the $E_{8}$ lattice. The automorphism group of the Leech lattice is Conway's group $\mathrm{Co}_{0}$, which is a double cover of the sporadic simple group $\mathrm{Co}_{1}$ (see [2]). In this paper I show how to write generators for $C o_{0}$, and many of its maximal subgroups, in terms of $3 \times 3$ octonion matrices (suitably interpreted).

We begin by summarising the notation and results of [12]. The octonions are an 8-dimensional real vector space, with basis $\left\{i_{t}: t \in P L(7)\right\}$, where $P L(7)=$ $\{\infty\} \cup \mathbb{F}_{7}$ is the projective line of order 7 , such that $i_{\infty}=1$ and the multiplication is given by the images under the subscript permutations $t \mapsto t+1$ and $t \mapsto 2 t$ of $i_{0} i_{1}=-i_{1} i_{0}=i_{3}$. The lattice $L$ is defined to be the copy of $E_{8}$ whose roots are $\pm i_{t} \pm i_{u}$ for $t \neq u$ and $\frac{1}{2} \sum_{t}\left( \pm i_{t}\right)$ with an odd number of minus signs. Let $s=\frac{1}{2}\left(-1+i_{0}+\cdots+i_{6}\right)$ and $R=\bar{L}$.

Writing $B=\frac{1}{2} L R$ we proved in [12] that $B$ is a copy of the $E_{8}$ lattice whose roots are $\pm i_{t}$ and the images under $t \mapsto t+1$ of $\frac{1}{2}\left( \pm 1 \pm i_{0} \pm i_{1} \pm i_{3}\right)$ and $\frac{1}{2}\left( \pm i_{2} \pm\right.$ $i_{4} \pm i_{5} \pm i_{6}$ ). Thus Coxeter's non-associative ring of integral octonions (see [5] and [3]) is $\frac{1}{2}\left(1+i_{0}\right) B\left(1+i_{0}\right)$, which is closed under multiplication. Moreover, using the Moufang laws we showed that $L \bar{s}=2 B$ and $B L=L$. The important content of [12] is the following definition. 
Definition 1 The octonionic Leech lattice $\Lambda=\Lambda_{\mathbb{O}}$ is the set of triples $(x, y, z)$ of octonions, with the norm $N(x, y, z)=\frac{1}{2}(x \bar{x}+y \bar{y}+z \bar{z})$, such that

(i) $x, y, z \in L$,

(ii) $x+y, x+z, y+z \in L \bar{s}$, and

(iii) $x+y+z \in L s$.

The main result of [12] is that $\Lambda$ is isometric to the Leech lattice.

\section{The monomial subgroup}

The reflection in any vector $r$ of norm 1 in the octonions can be expressed as the map

$$
x \mapsto-r \bar{x} r .
$$

In particular, since $1+i_{t}$ is perpendicular to $s$ we have $s=-\frac{1}{2}\left(1+i_{t}\right) \bar{s}\left(1+i_{t}\right)$. Using $R_{a}$ to denote the map $x \mapsto x a$, the Moufang law $((x a) b) a=x(a b a)$ can be expressed as $R_{a} R_{b} R_{a}=R_{a b a}$. In particular $R_{s}=-\frac{1}{2} R_{1+i_{t}} R_{\bar{s}} R_{1+i_{t}}$, which is equivalent to each of the following:

$$
\begin{aligned}
& R_{s} R_{-1+i_{t}}=R_{1+i_{t}} R_{\bar{s}} \\
& R_{-1+i_{t}} R_{s}=R_{\bar{s}} R_{1+i_{t}} .
\end{aligned}
$$

Combining two such relations gives

$$
R_{s} R_{1-i_{0}} R_{1+i_{t}}=-R_{1+i_{0}} R_{\bar{s}} R_{1+i_{t}}=R_{1+i_{0}} R_{1-i_{t}} R_{s}
$$

Therefore

$$
\begin{aligned}
\left(L\left(1-i_{0}\right)\right)\left(1+i_{t}\right) & =(L R) L=2 B L=2 L \\
\left(B\left(1-i_{0}\right)\right)\left(1+i_{t}\right) & =(B L) R=L R=2 B \\
\left((L s)\left(1-i_{0}\right)\right)\left(1+i_{t}\right) & =\left(\left(L\left(1+i_{0}\right)\right)\left(1-i_{t}\right)\right) s=2 L s .
\end{aligned}
$$

These three equations imply that the map $\frac{1}{2} R_{1-i_{0}} R_{1+i_{t}}$ acting simultaneously on all three coordinates preserves the octonion Leech lattice $\Lambda$.

Observe that the roots $1-i_{t}$ for $t=0,1,2,3,4,5,6$ form a copy of the root system of type $A_{7}$, whose Weyl group is the symmetric group $S_{8}$. Now the product of the reflections in $1-i_{0}$ and $1-i_{t}$ is the map

$$
x \mapsto \frac{1}{4}\left(1-i_{t}\right)\left(\left(1+i_{0}\right) x\left(1+i_{0}\right)\right)\left(1-i_{t}\right)
$$

which can be expressed as $\frac{1}{2} B_{1+i_{0}} \frac{1}{2} B_{1-i_{t}}$, where $B_{r}$ denotes the bi-multiplication map $x \mapsto r x r$. These elements act as 3 -cycles $(\infty, 0, t)$, so generate the rotation 
subgroup $A_{8}$ of the Weyl group. Finally we apply the triality automorphism which takes bimultiplications $B_{\bar{u}}$ by units $\bar{u}$ of norm 1 to right-multiplications $R_{u}$ by the octonion conjugate $u$, and deduce that the maps $\frac{1}{2} R_{1-i_{0}} R_{1+i_{t}}$ generate $2 \cdot A_{8}$, the double cover of $A_{8}$.

Adjoining the coordinate permutations and sign changes to this group gives a group $2 \cdot A_{8} \times S_{4}$. Adjoining also the symmetry $r_{0}:(x, y, z) \mapsto\left(x, y i_{0}, z i_{0}\right)$ described in [12] gives a group of shape $2^{3+12}\left(A_{8} \times S_{3}\right)$. The latter group is in fact a maximal subgroup of the automorphism group $2 \cdot \mathrm{Co}_{1}$ of the Leech lattice (see [11]), so we only need one more (non-monomial) symmetry to generate the whole of $2 \cdot \mathrm{Co}_{1}$.

\section{A complex reflection group}

In order to construct a non-monomial symmetry, we regard $s$ as a complex number $\frac{1}{2}(-1+\sqrt{-7})$ and consider the subset of the octonionic Leech lattice which lies inside the 3 -dimensional vector space over $\mathbb{Q}(s)=\mathbb{Q}(\sqrt{-7})$. This is a lattice spanned over $\mathbb{Z}[s]$ by the vectors $( \pm 2 s, 0,0),( \pm 2, \pm 2,0)$ and $\left( \pm s^{2}, \pm s, \pm s\right)$. Dividing through by $s$ we obtain a well-known lattice (see for example [4, p. $3]$ ) which has 42 vectors of norm 4 , and the 21 reflections in these vectors generate the automorphism group of the lattice, which is isomorphic to $2 \times L_{3}(2)$. More explicitly, this automorphism group is generated by the monomial subgroup $2^{3}: S_{3} \cong 2 \times S_{4}$ together with the matrix

$$
\frac{1}{2}\left(\begin{array}{ccc}
0 & \bar{s} & \bar{s} \\
s & -1 & 1 \\
s & 1 & -1
\end{array}\right),
$$

which is the negative of reflection in $(s, 1,1)$.

Now this matrix represents the map

$$
(x, y, z) \mapsto \frac{1}{2}((y+z) s, x \bar{s}-y+z, x \bar{s}+y-z)
$$

on the given complex vector space. But this can also be interpreted as a map on triples $(x, y, z)$ of octonions. We show next that, with this interpretation, it is also a symmetry of the octonion Leech lattice. To prove this claim, it is useful first to prove the following lemma.

Lemma $1(x, y, z) \in \Lambda$ if and only if the following three conditions hold:

(i) $x \in L$;

(ii) $x+y \in L \bar{s}=2 B$;

(iii) $x \bar{s}+y+z \in 2 L$. 
Proof. We use repeatedly the properties $2 L \subset L s \subset L$ and $2 L \subset L \bar{s} \subset L$. Suppose the three conditions of the lemma hold. Then $y=(x+y)-x \in L$ so $z=(x \bar{s}+y+z)-y-x \bar{s} \in L$. Also $y+z=(x \bar{s}+y+z)-x \bar{s} \in L \bar{s}$, and therefore $x+z=(x+y)-(y+z)+2 z \in L \bar{s}$. Finally $x+y+z=(x \bar{s}+y+z)+x s+2 x \in L s$.

Conversely if $(x, y, z)$ satisfies the conditions of the original definition, then $x \bar{s}+y+z=(x+y+z) \bar{s}+(y+z) s+2(y+z) \in 2 L$.

For convenience, write $\left(x^{\prime}, y^{\prime}, z^{\prime}\right)$ for the image of $(x, y, z)$ under the octonion map given by (1), and note that $s=\frac{1}{2}(-1+\sqrt{-7})$ satisfies $s^{2}+s+2=0$, so that $s+\bar{s}=-1, s^{2}=-2-s=\bar{s}-1$ and $\bar{s}^{2}=-2-\bar{s}=s-1$. Now we compute

(i) $x^{\prime}=\frac{1}{2}(y+z) s \in L$;

(ii) $x^{\prime}+y^{\prime}=\frac{1}{2}(x \bar{s}+y(-1+s)+z(1+s))=\frac{1}{2}(x+y \bar{s}-z) \bar{s} \in L \bar{s}$, using the fact that $\Lambda$ is invariant under coordinate permutations and sign-changes;

(iii) $x^{\prime} \bar{s}+y^{\prime}+z^{\prime}=(y+z)+x \bar{s} \in 2 L$;

and the claim is proved.

We summarise our results in the following theorem.

Theorem 1 The full automorphism group $2 \cdot \mathrm{Co}_{1}$ of the Leech lattice is generated by the following symmetries:

(i) an $S_{3}$ of coordinate permutations;

(ii) the map $r_{0}:(x, y, z) \mapsto\left(x, y i_{0}, z i_{0}\right)$;

(iii) the maps $\frac{1}{2} R_{1-i_{0}} R_{1+i_{t}}$ for $t=1,2,3,4,5,6$;

(iv) the $\operatorname{map}(x, y, z) \mapsto \frac{1}{2}((y+z) s, x \bar{s}-y+z, x \bar{s}+y-z)$.

\section{The normaliser of the complex reflection group}

We have described the subgroup $2 \cdot A_{8}$ generated by the maps $\frac{1}{2} R_{1-i_{0}} R_{1+i_{t}}$ as the double cover of the group of even permutations of $\{\infty, 0,1,2,3,4,5,6\}$. The stabiliser of $\infty$ is a subgroup $2 \cdot A_{7}$ generated by the elements $\frac{1}{2} R_{i_{1}-i_{0}} R_{i_{t}-i_{0}}$ for $t=2,3,4,5,6$. Since the roots $i_{t}-i_{0}$ are perpendicular to $s$, we know that $\left(i_{t}-i_{0}\right) \bar{s}\left(i_{t}-i_{0}\right)=-2 s$ and therefore $R_{i_{t}-i_{0}} R_{s}=R_{\bar{s}} R_{i_{t}-i_{0}}$. It follows that this group $2 \cdot A_{7}$ commutes with the reflection group $2 \times L_{3}(2)$ just described, giving rise to a subgroup $L_{3}(2) \times 2 \cdot A_{7}$.

It is well-known (see [11]) that this group has index 2 in a maximal subgroup of shape $\left(L_{3}(2) \times 2 \cdot A_{7}\right) .2$. To obtain the latter group we may adjoin an element such as $\frac{1}{2} R_{i_{0}-i_{1}} R_{s}^{*}$, where

$$
R_{s}^{*}=R_{s} \frac{1}{2}\left(\begin{array}{ccc}
s & 1 & 1 \\
1 & s & 1 \\
1 & 1 & s
\end{array}\right)
$$


Using the fact that $R_{i_{1}-i_{0}} R_{s}=R_{\bar{s}} R_{i_{1}-i_{0}}$ we can re-write this element in various ways such as

$$
\frac{1}{2} R_{\bar{s}} R_{i_{0}-i_{1}} \frac{1}{2}\left(\begin{array}{ccc}
s & 1 & 1 \\
1 & s & 1 \\
1 & 1 & s
\end{array}\right)=\frac{1}{2}\left(\begin{array}{ccc}
\bar{s} & 1 & 1 \\
1 & \bar{s} & 1 \\
1 & 1 & \bar{s}
\end{array}\right) \frac{1}{2} R_{\bar{s}} R_{i_{0}-i_{1}} .
$$

In particular, it squares to minus the identity.

We still have to show that this element is a symmetry of the octonionic Leech lattice. Writing $\left(x^{\prime}, y^{\prime}, z^{\prime}\right)$ for the image of $(x, y, z)$ under this map, we have

(i) $x^{\prime}=\frac{1}{4}((x \bar{s}+y+z) \bar{s})\left(i_{0}-i_{1}\right) \in \frac{1}{4}(2 L \bar{s}) L=\frac{1}{2}(L R) L=B L=L$ and by symmetry also $y^{\prime}, z^{\prime} \in L$;

(ii) $s^{2}-s=s(s-1)=s \bar{s}^{2}=2 \bar{s}$ and therefore $x^{\prime}-y^{\prime}=\frac{1}{2}\left((x-y)\left(i_{0}-i_{1}\right)\right) \bar{s} \in$ $\frac{1}{2}((L R) L) \bar{s}=L \bar{s}$ and again by symmetry $y^{\prime}-z^{\prime} \in L \bar{s}$;

(iii) $s+2=-s^{2}$ and so $x^{\prime}+y^{\prime}+z^{\prime}=\frac{1}{4}\left(((x+y+z) \bar{s})\left(i_{1}-i_{0}\right)\right) s^{2} \in \frac{1}{2}(L R) s^{2}=L s$.

Since, as remarked in [12], we can change signs arbitrarily in the definition, we have shown that the given element is a symmetry of the lattice.

Finally let us consider the action of $\frac{1}{2} R_{i_{0}-i_{1}} R_{s}^{*}$ by conjugation on $L_{3}(2) \times$ $2 \cdot A_{7}$. Since the factor $2 \cdot A_{7}$ commutes with the action of any matrix over $\mathbb{Q}(s)$, it follows easily that our element acts on the $2 \cdot A_{7}$ factor as the transposition $(0,1)$. Similarly, $R_{i_{0}-i_{1}}$ acts as complex conjugation $(s \leftrightarrow \bar{s})$ so our element acts on the $L_{3}(2)$ factor as complex conjugation followed by conjugation by the matrix $\left(\begin{array}{ccc}s & 1 & 1 \\ 1 & s & 1 \\ 1 & 1 & s\end{array}\right)$. More concretely, it commutes with the $S_{3}$ of coordinate permutations and maps the sign change on the last two coordinates (that is, the negative of reflection in $(2,0,0))$ to the negative of reflection in $(s, 1,1)$.

\section{The Suzuki chain}

The so-called Suzuki chain of subgroups of $2 \cdot \mathrm{Co}_{1}$ is a series of subgroups of the following shapes:

$$
\begin{array}{rll}
2 \cdot A_{9} & \times & S_{3} \\
2 \cdot A_{8} & \times & S_{4} \\
\left(2 \cdot A_{7}\right. & \times & \left.L_{3}(2)\right) .2 \\
\left(2 \cdot A_{6}\right. & \times & \left.U_{3}(3)\right) .2 \\
\left(2 \cdot A_{5}\right. & \circ & \left.2 \cdot J_{2}\right) .2 \\
\left(2 \cdot A_{4}\right. & \circ & \left.2 \cdot G_{2}(4)\right) .2 \\
& & 6 \cdot S u z .2
\end{array}
$$


We have already described two groups in this list, namely $2 \cdot A_{8} \times S_{4}$ and $\left(2 \cdot A_{7} \times L_{3}(2)\right) .2$. To obtain $2 \cdot A_{9} \times S_{3}$, we take the $S_{3}$ of coordinate permutations, together with the group $2 \cdot A_{8}$ which is generated by $\frac{1}{2} R_{1-i_{0}} R_{1+i_{t}}$, and extend $2 \cdot A_{7}$ to $2 \cdot S_{7}$ by adjoining the element $\frac{1}{2} R_{i_{0}-i_{1}} R_{s}^{*}$ as above. The map onto $A_{9}$ permuting the points $\{*, \infty, 0,1,2,3,4,5,6\}$ is then given by mapping $R_{1-i_{0}} R_{1+i_{t}}$ onto the 3 -cycle $(\infty, 0, t)$, as we have already seen, and mapping the extra element to $(*, \infty)(0,1)$. In terms of the root system of $L$, the factor $R_{s}^{*}$ of the new element corresponds to the root $s$, and extends the root system of type $\mathrm{A}_{7}$ spanned by $1-i_{t}$ to one of type $\mathrm{A}_{8}$.

To obtain the remaining groups in the Suzuki chain, all we have to do is adjoin to the complex reflection group $2 \times L_{3}(2)$ the part of $2 \cdot A_{9}$ which commutes with the appropriate subgroup $2 \cdot A_{n}$.

Consider first the subgroup $2 \cdot A_{6}$ generated by $\frac{1}{2} R_{i_{1}-i_{2}} R_{i_{1}-i_{t}}$ for $t=3,4,5,6$. This subgroup centralizes $\frac{1}{2} R_{1-i_{0}} R_{s}^{*}$ as well as the complex reflection group $2 \times$ $L_{3}(2)$. Together these generate the full centralizer $2 \times U_{3}(3)$, and by adjoining $\frac{1}{2} R_{1-i_{0}} R_{i_{1}-i_{2}}$ we obtain the whole group $\left(2 \cdot A_{6} \times U_{3}(3)\right) .2$, which is a maximal subgroup of $2 \cdot \mathrm{Co}_{1}$. An alternative generating set may be obtained by observing that the monomial subgroup of $U_{3}(3)$ is $4^{2}: S_{3}$ generated by $r_{0}:(x, y, z) \mapsto\left(x, y i_{0}, z i_{0}\right)$ and the coordinate permutations. Then we need only adjoin the element (1) to obtain $U_{3}(3)$. To prove that the group is indeed $U_{3}(3)$, first observe that all the generating matrices are written over $\mathbb{Q}\left(i_{0}, s\right)$, which is an associative ring of quaternions. Therefore these matrices define a quaternionic representation of the group in the usual sense, and it then suffices to reduce the representation modulo 3. Since both $i_{0}$ and $s-\bar{s}=\sqrt{-7}$ map to $\pm i$ in the field $\mathbb{F}_{9}=\mathbb{F}_{3}(i)$, it follows immediately that the generators map to unitary matrices. Notice that this is essentially the same description of the group $2 \times U_{3}(3)$ as Cohen's description of it as a quaternionic reflection group [1]. The reflecting vectors are, up to left quaternion scalar multiplication, the $3+12+48=63$ images under the monomial group of the Leech lattice vectors $(2 s, 0,0),(2,2,0)$ and $\left(s^{2}, s, s\right)$.

The next case may be obtained by taking the subgroup $2 \cdot A_{5}$ generated by $\frac{1}{2} R_{i_{2}-i_{3}} R_{i_{2}-i_{t}}$ for $t=4,5,6$. To extend from $U_{3}(3)$ to $2 \cdot J_{2}$ we may adjoin $\frac{1}{2} R_{1-i_{0}} R_{1+i_{1}}$, or alternatively extend the monomial subgroup from $4^{2}: S_{3}$ to $2^{3+4}: S_{3}$ by adjoining $r_{1}:(x, y, z) \mapsto\left(x, y i_{1}, z i_{1}\right)$. Then the involution centralizer $2^{2+4} A_{5}$ is generated by the diagonal symmetries, the coordinate permutation $(2,3)$, and the element $\frac{1}{2} R_{1-i_{0}} R_{s}^{\prime}$, where

$$
R_{s}^{\prime}=\left(\begin{array}{ccc}
s & 0 & 0 \\
0 & 1 & 1 \\
0 & 1 & -1
\end{array}\right)
$$

This is reminiscent of, but rather different from, the description of $2 \cdot J_{2}$ as a quaternionic reflection group in $[1,7,8]$.

The group $2 \cdot\left(A_{4} \times G_{2}(4)\right) .2$ is perhaps best described by taking the subgroup 
$2 \cdot A_{4}$ generated by $\frac{1}{2} R_{i_{0}-i_{3}} R_{i_{5}-i_{6}}$ and $\frac{1}{2} R_{i_{3}-i_{5}} R_{i_{5}-i_{6}}$. With the quaternionic labelling

\begin{tabular}{ll}
\hline 1 & 1 \\
$k$ & $j$ \\
$i$ & $k$ \\
$j$ & $i$ \\
\hline
\end{tabular}

given in [10] of each brick of the MOG (itself originally described in [6]), these elements become left-multiplication by $k$ and $-\omega$ respectively. As generators for $2 \cdot G_{2}(4)$ we may take those given above for $L_{3}(2)$, together with the monomial elements $\operatorname{diag}\left(1, i_{t}, i_{t}\right)$ for $t=1,2,4$, and the elements $\frac{1}{2} R_{1-i_{1}} R_{i_{2}-i_{4}}$ (equivalent to right multiplication by $k$ ) and $\frac{1}{2} R_{i_{1}-i_{2}} R_{i_{2}-i_{4}}$ (equivalent to right multiplication by $-\omega)$, as well as $\frac{1}{2} R_{1-i_{1}} R_{s}^{*}$.

The last case $6 \cdot S u z$ is of particular interest. It may be taken as the centralizer of the element $\frac{1}{2} R_{i_{3}-i_{5}} R_{i_{5}-i_{6}}$ of order 6 . Then it is generated by the sign-changes and coordinate permutations, together with the matrix of reflection in $(s, 1,1)$, and $\frac{1}{2} R_{1-i_{0}} R_{1+i_{t}}$ for $t=1,2,4$, as well as $\frac{1}{2} R_{1-i_{1}} R_{s}^{*}$. This extends to $6 \cdot S u z: 2$ by adjoining $\frac{1}{2} R_{1-i_{0}} R_{i_{5}-i_{6}}$.

\section{Some 2-local subgroups}

We have already seen how to generate the maximal 2-local subgroup of $C o_{0}$ which has shape $2^{3+12}\left(A_{8} \times S_{3}\right)$, acting monomially on the three octonion coordinates.

The involution centraliser has the shape $2^{1+8 \cdot} W\left(\mathrm{E}_{8}\right)^{\prime}$. If we take our involution to be $r_{0}^{2}=\operatorname{diag}(1,-1,-1)$, then the normal subgroup $2^{1+8}$ is generated by $r_{t}=\operatorname{diag}\left(1, i_{t}, i_{t}\right)$ for all $t$. Modulo this, the group $2 \cdot A_{8}$ together with $\operatorname{diag}\left(i_{t}, i_{t}, 1\right)$ generate a maximal subgroup of $W\left(\mathrm{E}_{8}\right)^{\prime}$, which may be extended to the whole group by adjoining an element such as $\frac{1}{2} R_{1-i_{0}} R_{s}^{\prime}$, where $R_{s}^{\prime}$ is as defined above.

Another maximal 2-local subgroup of $\mathrm{Co}_{0}$ is $2^{12} M_{24}$. This intersects our monomial group $2^{3+12}\left(A_{8} \times S_{3}\right)$ in a group

$$
2^{12} \cdot 2^{6}\left(S_{3} \times L_{3}(2)\right) \cong 2^{3+12} \cdot\left(2^{3} L_{3}(2) \times S_{3}\right) .
$$

To make the latter group, take the normal subgroup $2^{3+12} S_{3}$ generated by the coordinate permutations and all $r_{t}$, and the subgroup of $2 A_{8}$ generated by

$$
\frac{1}{8} R_{1-i_{2}} R_{i_{3}-i_{5}} R_{i_{4}-i_{1}} R_{i_{6}-i_{0}} R_{1-i_{1}} R_{i_{2}-i_{4}}
$$

and its images under $i_{t} \mapsto i_{t+1}$. Now let $\pi$ be the coordinate permutation $(1,2)$, and adjoin the element

$$
r_{1}^{\pi R_{1-i_{1}} R_{s}^{\prime} / 2}=\frac{1}{4} R_{1-i_{1}} R_{s}^{\prime} \operatorname{diag}\left(i_{1}, 1, i_{1}\right) R_{1-i_{1}} R_{s}^{\prime} .
$$


A little calculation shows that this element lies in the subgroup $2^{12} M_{24}$ which acts monomially on the MOG described above, but does not lie in $2^{3+12}\left(A_{8} \times S_{3}\right)$. Hence we have obtained generators for $2^{12} M_{24}$.

A similar process gives generators for the other 2-constrained maximal 2local subgroup $2^{5+12}\left(S_{3} \times 3 S_{6}\right)$. In this case we adjoin the above element to the subgroup $2^{3+12}\left(\left(A_{4} \times A_{4}\right) .2 \times S_{3}\right)$ obtained by restricting to the subgroup of $2 \cdot A_{8}$ generated by $\frac{1}{2} R_{1-i_{1}} R_{1+i_{2}}, \frac{1}{2} R_{1-i_{1}} R_{1+i_{4}}, \frac{1}{2} R_{i_{0}-i_{3}} R_{i_{0}-i_{6}}, \frac{1}{2} R_{i_{0}-i_{3}} R_{i_{0}-i_{5}}$ and $\frac{1}{4} R_{1-i_{0}} R_{i_{1}-i_{3}} R_{i_{2}-i_{6}} R_{i_{4}-i_{5}}$.

\section{References}

[1] A. M. Cohen, Finite quaternionic reflection groups, J. Algebra 64 (1980), 293-324.

[2] J. H. Conway, A group of order 8,315,553,613,086,720,000, Bull. London Math. Soc. 1 (1969), 79-88.

[3] J. H. Conway and D. A. Smith, On quaternions and octonions: their geometry, arithmetic and symmetry, A. K. Peters (2003).

[4] J. H. Conway, R. T. Curtis, S. P. Norton, R. A. Parker and R. A. Wilson, An Atlas of Finite Groups, Clarendon Press, Oxford, 1985.

[5] H. S. M. Coxeter, Integral Cayley numbers, Duke Math. J. 13 (1946), 567578.

[6] R. T. Curtis, A new combinatorial approach to $M_{24}$, Math. Proc. Cambridge Philos. Soc. 79 (1976), 25-42.

[7] J. Tits, Quaternions over $\mathbb{Q}(\sqrt{ } 5)$, Leech's lattice and the sporadic group of Hall-Janko, J. Algebra 63 (1980), 56-75.

[8] R. A. Wilson, The geometry of the Hall-Janko group as a quaternionic reflection group, Geom. Dedicata 20 (1986), 157-173.

[9] R. A. Wilson, The complex Leech lattice and maximal subgroups of the Suzuki group, J. Algebra 84 (1983), 151-188.

[10] R. A. Wilson, The quaternionic lattice for $2 G_{2}(4)$ and its maximal subgroups J. Algebra 77 (1982), 449-466.

[11] R. A. Wilson, The maximal subgroups of Conway's group Co $o_{1}, J$. Algebra 85 (1983), 144-165.

[12] R. A. Wilson, Octonions and the Leech lattice, J. Algebra 322 (2009), 21862190. 